\title{
TURBULENCE IN STELLAR INTERIORS
}

\section{JEAN-PAUL ZAHN ${ }^{1,2}$}

${ }^{1}$ Observatoire Midi-Pyrénées, 14 ave. E. Belin, 31400 Toulouse, France

${ }^{2}$ Astronomy Department, Columbia University, New York, NY 10027, U.S.A.

ABSTRACT This review focusses on the most recent work which has been achieved concerning turbulence in stellar interiors. Among all possible causes for such turbulence, the most powerful is certainly the convective instability in unstably stratified regions, but little was known until now beyond the fact that thermal convection is capable of establishing an almost adiabatic stratification. The latest numerical simulations (Stein \& Nordlund 1989; Cataneo et al. 1991) show that turbulent convection is highly intermittent, with strong downdrafts carrying most of the energy flux.

These plumes penetrate in the stable region below, which they render nearly adiabatic over some distance (Zahn 1991); the penetration depth should be measured soon through acoustic sounding of the Sun (Berthomieu et al. 1992). Such plumes are likely to exist also in a convective core, and to penetrate into the radiative region above (Roxburgh 1989).

Another type of turbulence apparently occurs in the radiation zones, due to differential rotation. It is much weaker, and strongly anisotropic, with more vigorous motions in the horizontal directions than in the vertical. Its role is negligible in the heat transport, but it acts to suppress the latitudinal variation of the angular velocity. This anisotropic turbulence also interfers with the meridian circulation, and it partly inhibits the advection of chemical elements (Chaboyer \& Zahn 1992). The strongest indication so far for such turbulence is the thinness of the solar tachocline (Spiegel \& Zahn 1992): this boundary layer, in which the rotation changes from differential in the convection zone to uniform below, has been detected through helioseismology, but it is still unresolved (Goode et al. 1991).

Assuming that such anisotropic turbulence enforces throughout the radiation zone a rotation state in which the angular velocity depends mainly on depth, it is possible to derive an explicit expression for the meridian flow, and thus to describe the evolution in time of the interior rotation (Zahn 1992). The main result is that both the meridian circulation and the turbulence are determined by the rate at which the star loses angular momentum. When there is no such loss, the meridian flow is very weak, and it can even vanish in slow rotators.

Keywords: Convection, convective penetration; meridian circulation; turbulence. 


\section{TURBULENT CONVECTION}

Until recently, turbulence in stellar interiors has been synonymous with thermal convection, which indeed is the strongest of all dynamical instabilities, once the star has settled in hydrostatic equilibrium. Its contribution to the heat transport enforces a nearly adiabatic temperature profile, which is implemented in our models of stellar structure. We do this in general by resorting to the mixing-length treatment, for lack of a better prescription. And some of us still try to improve on that approach, with more or less sophistication.

But another, more promising road has been opened thanks to numerical simulation. The computers have progressed to a point which allows threedimensional calculations, with a resolution of about $100^{3}$. This is still insufficent to encompass a whole convection zone, and to correctly represent the kinetic energy cascade, but it permits to explore the organization of the motions on the largest scales. These resemble very much the granular pattern on the surface of the Sun, and their spectroscopic signature also agrees well with the observations (Nordlund \& Dravins 1990).

Another striking result of these computer experiments is the presence of strong, long-lived downdrafts (Chan \& Sofia 1986; Stein \& Norlund 1989; Cattaneo et al. 1991). These behave like the thermal plumes which are well known in atmospheric sciences: they entrain some of the surrounding matter, and their life-time is much longer than the time the flow takes to travel through them. Such downdrafts were detected already in earlier two-dimensional calculations (Hulburt etal. 1984). They originate below the upper boundary layer, which is turbulent in the classical sense (the life-time of the eddies is of the order of their turn-over time). The plumes cross the whole computational domain, which is still limited by the possibilities of the present computers, but one can anticipate that some of them would traverse the whole convection zone. The picture which emerges today is sketched in fig. 1.

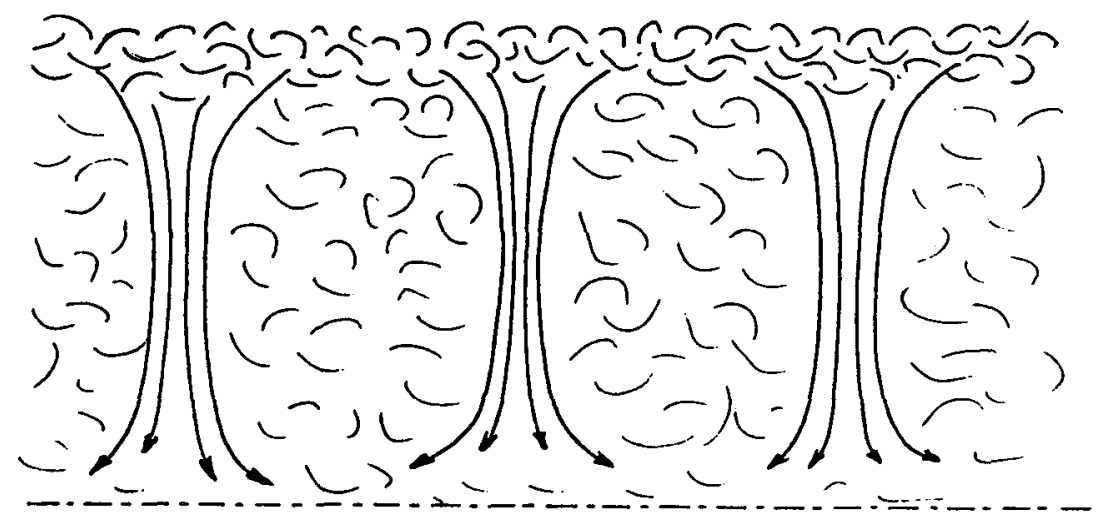

Fig. 1. A plausible picture of the convective envelope in solar-like stars, with downwards directed plumes traversing the whole convection zone. The dash-dotted line delineates the nearly adiabatic domain, including the region of convective penetration (see below). 
Those plumes carry kinetic energy downwards and thermal energy (enthalpy) upwards, and surprisingly the two seem to cancel each other (Cattaneo et al. 1991). Therefore, it is the much quieter intersticial medium which is responsible for conveying the net energy flux to the surface. It remains to examine the full implications of this highly intermittent picture of the turbulent convection zone, both on the transport of heat and on that of angular momentum (cf. Rieutord \& Zahn 1991; Simon \& Weiss 1991).

Let us mention that such plumes are also observed in laboratory expermients, where the stratification is very weak: there they are directed either upwards or downwards, with a prefernce for the direction which allows for penetrative convection (cf. Whitehead \& Chen 1970).

\section{CONVECTIVE PENETRATION}

Penetrative convection, or overshooting as it is sometimes called by astrophysicists, has been the subject of a long lasting debate (see Renzini 1987). However, there is overwhelming evidence for such penetration, in geophysical fluids, in the laboratory, and now also in the numerical simulations: the convective motions do not stop abruptly at the edge of the unstable region, but they travel beyond over some distance, and continue to enforce a nearly adiabatic stratification. The question is thus not whether penetration does occur in stellar interiors, but how important it is.

Stellar models that include this effect most often postulate a penetration depth of a fraction of the pressure scale height, or else of the radius of the convective core. And this fraction is calibrated by comparing the theoretical predictions with the observations. But everyone agrees that there is a need for a better prescription, on a sound physical basis.

It is appropriate to seek an answer in numerical simulations, like those which have been mentioned above, but with boundary conditions that allow for penetration. Those which have been performend in two dimensions show indeed that the conspicuous downdrafts penetrate rather deep into the stable region (Hurlburt et al. 1986). But the computer power which is presently available does not permit yet to explore the relevant parameter range. The goal is even more remote in three dimensions, although some attempts have been made already at low resolution (Brandenburg et al. 1991; Cattaneo, private communication).

In the meanwhile, one must make up with some reasonable prediction, based on minimal and plausible assumptions. One has been proposed by Roxburgh $(1978,1989)$, but its validity has been questioned because it rests on the assumption that the convective motions are confined in a nearly adiabatic domain (Baker \& Kuhfuß 1987). This assumption therefore implies that the penetrative motions are efficient enough to establish such a temperature profile in the stable adjacent region. It can be shown that this requirement is met in a convective core, provided that rising, plume-like motions occur there, as it is suggested indeed by laboratory experiments (Zahn 1991).

Roxburgh's criterion states that the following integral vanishes at the edge of the penetration region:

$$
\int\left(L_{n u c}-L_{r a d}\right) d\left(\frac{1}{T}\right)=0
$$


where $T$ is the temperature, $L_{n u c}$ the total energy flux produced by the nuclear reactions, and $L_{\text {rad }}$ the total flux carried by radiation in the nearly adiabatic temperature stratification. This predicts a penetration depth which is a fraction of about $20 \%$ of the core radius, and which increases with its mass (Roxburgh 1992; see also Zahn 1991). The effect of neglecting the viscous dissipation is now being investigated (see the poster by Roxburgh \& Simmons, this meeting).

Unfortunately, this integral constraint cannot be employed for a convective envelope, because the departures from adiabaticity are too large at the surface boundary. One then needs to postulate the form of the convective motions, and the most natural assumption is that they are organized in plumes, as described above. This was first done by Schmitt et al. (1984), with application to the base of the solar convection zone. They found empirically that the penetration depth scales as $f^{1 / 2} W_{i}^{3 / 2}$, with $W_{i}$ being the velocity at the top of the subadiabatic region and $f$ a measure of the area occupied by the plumes.

The same scaling can be derived theoretically, as was shown by Zahn (1991). Furthermore, with a plausible estimate of the initial velocity $W_{i}$, one finds that the penetration depth is of the order of the scale height of the radiative conductivity: $|d r / d \ln \chi|\left(\chi=16 \sigma T^{3} / 3 \rho \kappa\right.$ with the usual notations). Thus the penetration depth $L_{p}$ is given by

$$
\frac{L_{p}}{H_{P}}=\frac{\zeta}{\chi_{P}}
$$

where $H_{P}$ is the pressure scale-height and $\chi_{P}=(\partial \log \chi / \partial \log P)_{a d}$. The unknown parameter $\zeta$ is related to the third moment of the convective velocity: it should be of order unity, but is presumably a little less. Once it has been calibrated with the Sun, it can be used in other stellar models with a deep convective envelope, much as one does with the familiar mixing-length parameter $\alpha$.

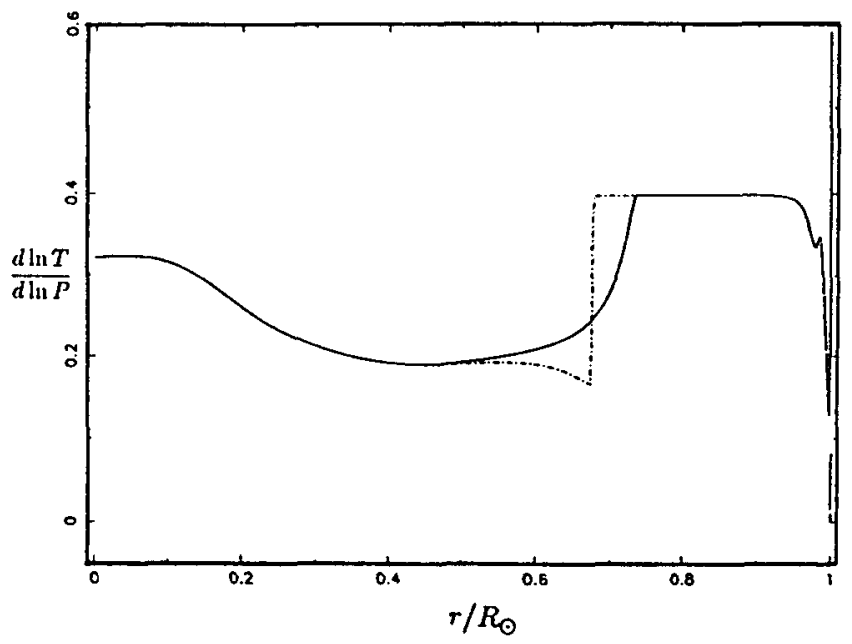

Fig. 2. The logarithmic temperature gradient in a standard solar model (continuous line), compared with a model allowing for a convective penetration of $30,000 \mathrm{~km}$ (dash-dotted line). Note the discontinuity at the edge of the penetration region (Berthomieu et al. , this meeting). 
This calibration has been undertaken by Berthomieu et al. (1992). A series of solar models have been computed with the code CESAM built at the Nice Observatoire (see Morel etal. 1990); they include an increasing amount of penetration at the base of the convection zone, the parameter $\zeta$ varying from 0 to 1 . These models were evolved from a ZAMS homogeneous model to the present age of the Sun, where they were fitted to the observed radius and luminosity. One of such models is depicted in fig. 2. The comparison of the eigenfrequencies between these models and the Sun (Libbrecht et al. 1990) is used to determine the value of the penetration parameter $\zeta$. Work is still in progress, in order to include all relevant physical processes; presently, the best estimate is around 0.70 , which corresponds to a penetration depth of about $20,000 \mathrm{~km}$ below the solar convection zone.

\section{THE SOLAR TACHOCLINE}

Thanks to helioseismology, it is now possible to probe the distribution of the angular velocity as a function of radius and latitude. The surface rotation, with its equatorial acceleration, carries on to the bottom of the convection zone with little depth dependence. In the radiation zone below, the equatorial and polar rotation rates approach a common value, within a layer which is too thin to be resolved as yet, i.e. which is less than about 1/10 of the solar radius (Brown et al. 1989; Goode et al. 1991).

The theory of this transition layer has been completed recently by Spiegel and Zahn (1992). Considering the observed rotation law in the convection zone as given, they predict the angular velocity in the radiative interior below by expressing the hydrostatic balance and the conservation of energy. The governing equations are simplified by approximations usually applied to thin layers with rapid rotation. A similar approach is taken by the oceanographers, when they study ocean circulation driven by the wind stress at the sea surface: for that reason the solar transition layer has been named tacholine.

The velocity boundary conditions applied to the top of the radiation enforce a horizontal pressure gradient, leading in turn to a horizontal temperature gradient in the pole-equator direction. This strong link between the temperature and differential rotation is well known in geophysical fluid dynamics as the thermal wind. In the Sun, because of radiation, the horizontal temperature distribution diffuses downwards, and it is accompanied by the differential rotation, which, in the inviscid case, can be modified only by advecting angular momentum.

But the meridian motions required for this are hindered by the stable stratification, and thus the transition layer spreads inward at a much slower rate. Instead of growing in time as in the normal diffusive way like $t^{1 / 2}$, and the thickness $h$ of the layer increases approximately as

$$
h \approx R_{c}\left(t / t_{E S}\right)^{1 / 4}
$$

where the local Eddington-Sweet time is given by $t_{E S}=(\Omega / N)^{-2} R_{c}^{2} / K$, in terms of the mean rotation rate $\Omega$, the buoyancy frequency $N$, and $K$ the thermal diffusivity. A similar temporal evolution is found in the stratified spin-down problem (e.g. Sakurai 1970; Spiegel 1972). For the Sun, $h \approx 210^{5} \mathrm{~km}$ after $4.610^{9} \mathrm{yrs}$, according to this crude formula. 


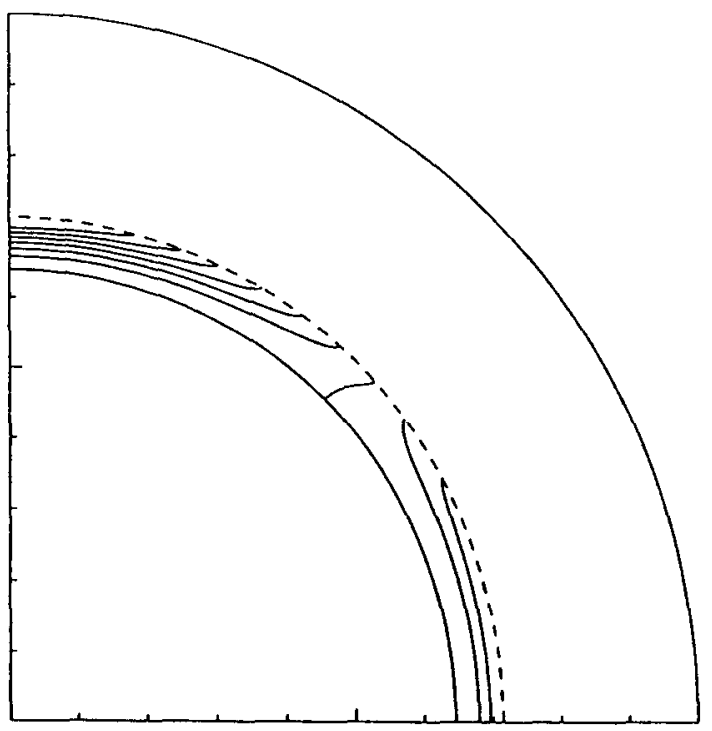

Fig. 3. The solar tachocline, whose thickness has been set arbitrarily to 50,000 $\mathrm{mk}$ (the actual value depends on the horizontal viscosity $\nu_{h}$ ). The continuous lines show the contours of the angular velocity. The interior rotation is that of the convection zone at the latitude $42^{\circ}$ (Spiegel \& Zahn 1992; courtesy Astr. Ap.).

The actual calculation, allowing for the variation of the rotation rate with time, yields an even larger result: $h \approx 310^{5} \mathrm{~km}$. Hence, we conclude that the differential rotation of the convection zone would have extended well into the Sun, if radiative diffusion were alone responsible for its spread. The apparent discontinuity represented by the observed tachocline must therefore be ascribed to another physical process, capable of preventing the expansion of the layer.

In deriving the estimate above, the flow has been treated implicitely as laminar, while it is a well established fact that, at sufficiently high Reynolds numbers, virtually any flow becomes turbulent. Thus there is little doubt that the horizontal shear of the differential rotation will be unstable (Zahn 1975). An enhanced isotropic viscosity would spread the tachycline even more quickly into the interior than before, but one verifies that with sufficiently anisotropic turbulence one can construct solutions that resemble the observed tachycline (fig. 3).

It thus proves the presence, below the solar convection zone, of a predominantly horizontal tubulence, such as encountered in the geophysical context (Rhines 1979). In this regime, the horizontal advection of angular momentum is balanced by the turbulent stresses, and a steady state is achieved in a layer whose thickness can be well within the bound set by observations. That thickness is determined by the turbulent Prandtl number, which is here the ratio between the horizontal eddy viscosity $\nu_{h}$ and the thermal diffusivity $K$ :

$$
h=R_{c}(\Omega / N)^{1 / 2}\left(K / \nu_{h}\right)^{1 / 4} .
$$


The theory also predicts that the interior uniform rotation is intermediate between the polar and equatorial rotation rates of the convection zone, in agreement with the observations. The vertical differential rotation changes sign at a latitude of about $42^{\circ}$ (see fig. 3).

\section{ROTATION-INDUCED TURBULENCE}

In the solar tachocline, the differential rotation which is responsible for the turbulent motions is enforced by the convection zone above. But it can also arise from the advection of angular momentum through a large scale circulation. The origin of such meridian circulation has been known since Eddington (1925) and Vogt (1925): due to the centrifugal force, the radiation flux is no longer conservative, and the star cannot achieve thermal equilibrium. A large scale advection is thus induced to preserve the conservation of energy.

This meridian flow depends sensitively on the rotation law, which itself is continually modified by the flow. The differential rotation is liable to various instabilities, as we have mentioned above, and the turbulent motions that then occur do also interfer with the transport of angular momentum. The problem to solve is thus rather intricate, and until recently, it had not been treated in a self-consistent way, mainly because of our poor knowledge of the turbulent transport.

However, significant progress can be achieved by assuming that the turbulence is highly anisotropic, as observed in the solar tachocline: then it instaures a rotation state in which the angular velocity depends little on latitude, and may be considered as varying only with depth (Zahn 1992). With such a shellular rotation law, it is possible to derive an explicit expression for the meridian velocity, $u(r, \theta)=U(r) P_{2}(\cos \theta)$, with $U(r)$ involving up to the third derivative of the angular velocity $\Omega(r)$ ( $r$ and $\theta$ are the polar coordinates).

The evolution of the rotation profile is governed by the conservation of angular momentum, which is then expressed by

$$
\frac{\partial}{\partial t}\left[\rho r^{2} \Omega\right]=\frac{1}{5 r^{2}} \frac{\partial}{\partial r}\left[\rho r^{4} \Omega U\right]+\frac{1}{r^{2}} \frac{\partial}{\partial r}\left[\rho \nu_{t} r^{4} \frac{\partial \Omega}{\partial r}\right],
$$

with the usual notations, and $\nu_{t}$ being the vertical component of the turbulent viscosity. This viscosity is due essentially to the shear in the vertical direction, and $\nu_{t}$ is proportional to $(\partial \Omega / \partial r)^{2}$ (Zahn 1975). A similar equation has been used by Endal \& Sofia (1976), and later by Pinsonneault et al. (1989) to model the transport of angular momentum in stellar interiors, but the advective term was lacking.

The p.d.e. above is of first order in time, and of fourth-order in space; it must be solved with suitable initial and boundary conditions, of which the most important expresses the loss of the angular momentum $J$ at the surface of the star:

$$
-\frac{1}{5} \rho r^{4} \Omega U-\rho \nu_{t} r^{4} \frac{\partial \Omega}{\partial r}=-\frac{3}{8 \pi} \frac{\partial J}{\partial t} .
$$

When the star is not subject to a wind, the rotation rate settles rapidly into a state with little meridian circulation, the latter just compensating the adverse flux of angular momentum due to the turbulent motions. Moreover, if 
the rotation speed lies below some threshold, the shear of the differential rotation is too weak to generate such turbulence, and there is no circulation at all.

The situation is drastically different when the star emits a wind. Then a bulk circulation sets in to carry the angular momentum from the interior to the surface, and its speed is governed by the rate of angular momentum loss. The rotation profile adjusts slavishly to produce the required meridian flow, and so does also the turbulence. The core of the star rotates substantially faster than the surface, and one may expect that this is the case, in particular, for the Sun (fig. 4).

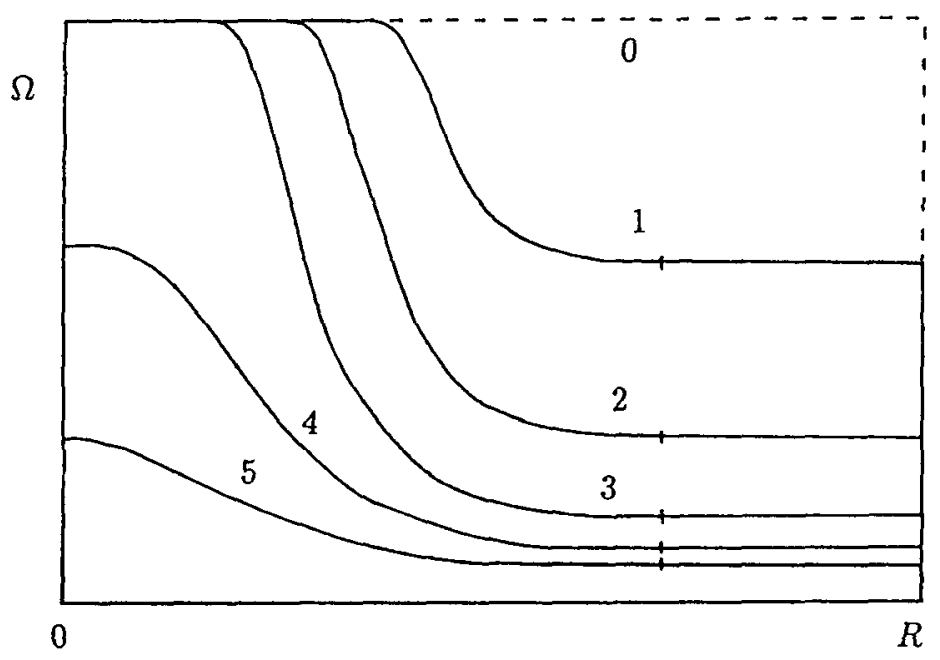

Fig. 4. Evolution of the rotation profile in a solar-like star which loses angular momentum (Zahn 1992). Work is in progress to refine this sketch.

This notion, that it is the spindown which drives the circulation, is well known for viscous fluids as the so- called Ekman pumping (Bondi \& Littleton 1948; Greenspan \& Howard 1963). Its relevance for stars has been recognized by Howard et al. (1967), with radiative diffusion taking the role of the viscous coupling, and with the whole convection zone acting as the turbulent Ekman layer (Bretherton \& Spiegel 1968). But for some reason the astronomical community seems to have ignored these pionneering works.

The transport of angular momentum is not much affected by the turbulent motions, whose role is chiefly to enforce the shellular rotation state. In contrast, the advection of chemical species is partly inhibited by the horizontal component $D_{h}$ of the turbulent diffusivity, which acts to smooth out the horizontal inhomogeneities that are built up by the circulation. When $D_{h} \geq r U$, the vertical transport of matter behaves like a diffusion, with an effective diffusivity given by:

$$
D_{\text {ess }}=\frac{1}{30} \frac{(r U)^{2}}{D_{h}}
$$

This result was established by Chaboyer and Zahn (1992), who showed that the process is closely related to Taylor's shear dispersion (Taylor 1953). Their 
predictions were confirmed by Charbonneau (1992), who actually simulated, with a two-dimensional code, the interference of anisotropic turbulence with a meridian flow.

This turbulent erosion provides a straightforward explanation for the strong link between the transport of lithium and that of angular momentum, and moreover for the disparity which is observed between the efficiencies of these transports in the case of the Sun (Law et al. 1984). With some simplifying assumptions, it is possible to show that the surface abundance of lithium varies approximately as

$$
-d \log c=-k \log J
$$

where $k$ is a constant that depends only on the location of the base of the convection zone and that of the lithium destruction layer, but not on the spindown law itself (Zahn 1992).

Work is in progress to explore all consequences of the master equation (5) above, and in particular to predict the internal rotation of the Sun.

\section{ACKNOWLEDGEMENTS}

Support for this work was provided by the U.S. Air Force under grant AFOSR 89-0012 to Columbia University, and by the Centre National de la Recherche Scientifique (GDR 131).

\section{REFERENCES}

Baker, N.H., KuhfuB, R. 1987, Astr. Ap., 185, 117

Berthomieu, G., Morel, P., Provost, J., Zahn, J.-P. 1992, Astr. Ap., (preprint)

Bondi, H., Lyttleton, R.A. 1948, Proc. Cambridge Phil. Soc., 44, 345

Brandenburg, A., Jennings, R., Nordlund, Á., Rieutord, M., Ruokilainene, J., Stein, R., Tuominen, I. 1992, Ap. J., (preprint)

Bretherton, F.P., Spiegel, E.A. 1968, Ap. J., 153, 277

Brown, T.M., Christensen-Dalsgaard, J., Dziembowski, W.A., Goode, P., Gough, D.O., Morrow, C.A., 1989, Ap. J., 343, 526

Cattaneo, F., Brummel, N.H., Toomre, J., Malagoli, A., Hurlburt, N.E. 1991, Ap. J., 370,282

Chaboyer, B., Zahn, J.-P. 1992, Astr. Ap., 253, 173

Chan, K.L., Sofia, S. 1986, Ap. J., 307, 222

Charbonneau, P. 1992, Astr. Ap., (preprint)

Eddington, A.S. 1925, Observatory, 48, 78

Endal, A.S., Sofia, S. 1976, Ap. J., 210, 184

Goode, P.R., Dziembowski, W.A., Korzennick, S.G., Rhodes, E.J. 1991, Ap. J., 367, 649

Greenspan, H., Howard, L.N. 1963, J. Fluid Mech., 17, 385

Howard, L.N., Moore, D.W., Spiegel, E.A. 1967, Nature, 214, 1297

Hurlburt, N.E., Toomre, J., Massaguer, J.M. 1984, Ap. J., 282, 557

Hurlburt, N.E., Toomre, J., Massaguer, J.M. 1986, Ap. J., 311, 563

Law, W.Y., Knobloch, E., Spruit, H.C. 1984, Observational Tests of Stellar Evolution Theory (ed. A. Maeder, A. Renzini; Reidel), 523

Libbrecht, K.G., Woodard, M.F., Kaufman, J.M. 1990, Ap. J. Suppl., 74, 1129

Morel, P., Provost, J., Berthomieu G. 1990, Solar Phys. 128, 7

Nordlund, Å., Dravins, D. 1990, Astr. Ap., 228, 155 
Rhines, P.B. 1979, Ann. Rev. Fluid Mech., 11, 401

Renzini, A. 1987, Astr. Ap., 188, 49

Rieutord, M., Zahn, J.-P. 1991, The Sun and Cool Stars: Activity, Magnetism, Dynamos (ed. I. Tuominen, D. Moss, G. Rüdiger; Springer), 193

Roxburgh, I.W. 1978, Astr. Ap., 65, 281

Roxburgh, I.W. 1989, Astr. Ap., 211, 361

Roxburgh, I.W. 1992, Astr. Ap., (preprint)

Sakurai, T. 1970, Stellar Rotation (ed. A. Slettebak; Reidel), 329

Schmitt, J.H.M.M., Rosner, R., Bohn, H.U. 1984, Ap. J., 282, 316

Simon, G.W., Weiss, N.O. 1991, M.N.R.A.S., 252, lp

Spiegel, E.A. 1972, Physics of the Solar System (ed. S.I. Rasool; NASA, Washington)

Spiegel, E.A., Zahn, J.-P. 1992, Astr. Ap., (preprint)

Spruit, H.C., Nordlund, A., Title, A.M. 1990, Ann. Rev. Astr. Ap., 28, 263

Stein, R., Nordlund, $\AA .1989, A p . J ., 242$, L95

Taylor, G.I. 1953, Proc. Roy. Astron. Soc. London A2, 19, 186

Vogt, H. 1925, Astron. Nachr., 223, 229

Whitehead, J.A., Chen, M.M. 1970, J. Fluid Mech., 40, 549

Zahn, J.-P. 1975, Mém. Soc. Roy. Sci. Liège, 6e série, 8, 31

Zahn, J.-P. 1991, Astr. Ap., 252, 179

Zahn, J.-P. 1992, Astr. Ap., (preprint) 